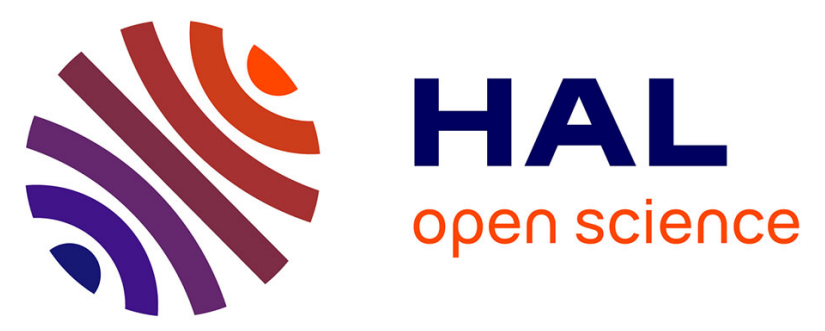

\title{
Earth's core and inner-core resonances from analysis of VLBI nutation and superconducting gravimeter data
}

\author{
S. Rosat, S.B. Lambert, C. Gattano, M. Calvo
}

\section{To cite this version:}

S. Rosat, S.B. Lambert, C. Gattano, M. Calvo. Earth's core and inner-core resonances from analysis of VLBI nutation and superconducting gravimeter data. Geophysical Journal International, 2017, 208

(1), pp.211 - 220. 10.1093/gji/ggw378 . hal-01397310

\section{HAL Id: hal-01397310 \\ https://hal.science/hal-01397310}

Submitted on 5 Jan 2022

HAL is a multi-disciplinary open access archive for the deposit and dissemination of scientific research documents, whether they are published or not. The documents may come from teaching and research institutions in France or abroad, or from public or private research centers.
L'archive ouverte pluridisciplinaire HAL, est destinée au dépôt et à la diffusion de documents scientifiques de niveau recherche, publiés ou non, émanant des établissements d'enseignement et de recherche français ou étrangers, des laboratoires publics ou privés. 


\title{
Earth's core and inner-core resonances from analysis of VLBI nutation and superconducting gravimeter data
}

\author{
S. Rosat, ${ }^{1}$ S.B. Lambert, ${ }^{2}$ C. Gattano ${ }^{2}$ and M. Calvo ${ }^{1,3}$ \\ ${ }^{1}$ Institut de Physique du Globe de Strasbourg, IPGS - UMR 7516, CNRS et Université de Strasbourg (EOST), 5 rue René Descartes, \\ F-67084 Strasbourg, France. E-mail: severine.rosat@unistra.fr \\ ${ }^{2}$ SYRTE, Observatoire de Paris, PSL Research University, CNRS, Sorbonne Universités, UPMC Univ. Paris 06, LNE, Paris, France \\ ${ }^{3}$ Observatorio Geofisico Central, Instituto Geogràfico Nacional (IGN), c/Alfonso XII 3, E-28014 Madrid, Spain
}

Accepted 2016 October 4. Received 2016 September 2; in original form 2016 June 24

\begin{abstract}
S U M MAR Y
Geophysical parameters of the deep Earth's interior can be evaluated through the resonance effects associated with the core and inner-core wobbles on the forced nutations of the Earth's figure axis, as observed by very long baseline interferometry (VLBI), or on the diurnal tidal waves, retrieved from the time-varying surface gravity recorded by superconducting gravimeters (SGs). In this paper, we inverse for the rotational mode parameters from both techniques to retrieve geophysical parameters of the deep Earth. We analyse surface gravity data from 15 SG stations and VLBI delays accumulated over the last $35 \mathrm{yr}$. We show existing correlations between several basic Earth parameters and then decide to inverse for the rotational modes parameters. We employ a Bayesian inversion based on the Metropolis-Hastings algorithm with a Markov-chain Monte Carlo method. We obtain estimates of the free core nutation resonant period and quality factor that are consistent for both techniques. We also attempt an inversion for the free inner-core nutation (FICN) resonant period from gravity data. The most probable solution gives a period close to the annual prograde term (or $S_{1}$ tide). However the 95 per cent confidence interval extends the possible values between roughly 28 and $725 \mathrm{~d}$ for gravity, and from 362 to $414 \mathrm{~d}$ from nutation data, depending on the prior bounds. The precisions of the estimated long-period nutation and respective small diurnal tidal constituents are hence not accurate enough for a correct determination of the FICN complex frequency.
\end{abstract}

Key words: Probability distributions; Time variable gravity; Earth rotation variations; Core, outer core and inner core.

\section{INTRODUCTION}

In a rotating elliptical Earth with a fluid core and a solid inner core, there exist four rotational normal modes: the Chandler wobble $(\mathrm{CW})$, the free core nutation $(\mathrm{FCN})$, the free inner-core nutation (FICN) and the inner-core wobble (ICW). The existence of the FICN is related to the presence of a slightly tilted and oblate solid inner core (IC) in the fluid outer core (de Vries \& Wahr 1991; Herring et al. 1991; Mathews et al. 1991). The misalignment of the rotation and figure axis of the IC with respect to the mantle induces fluid pressure acting on the elliptical inner-core boundary (ICB). Gravitational forces on the tilted IC also contribute to the internal torque (Dumberry 2009). Though, in a space-fixed reference frame, the FICN is prograde and the FCN is retrograde, in a terrestrial reference frame, the FCN has a nearly diurnal retrograde motion (for this reason, the latter is sometimes called nearly diurnal free wobble). The Earth's response to the tidal forcing leads to a resonance effect when the forcing frequency is close to the eigenfrequency of a normal mode. The resonance perturbs amplitudes and phases of the tidal waves observed by gravimetry and of the nutations observed by very long baseline interferometry (VLBI). In turn, the precise determination of the tidal and nutation amplitudes allows one to constrain the eigenfrequencies associated with the resonances, and, thereby, some geophysical parameters entering the expressions of the resonant frequencies.

While the resonance associated with the FCN has been clearly observed and analysed using VLBI measurements of nutation (e.g. Lambert \& Dehant 2007) or surface (gravity, strain, tilt, borehole water level) observations (see for instance Blum et al. 1973; Neuberg \& Zürn 1986; Zaske et al. 2000; Ducarme et al. 2007; Rosat et al. 2009; Amoruso et al. 2012) or both space nutation and surface gravity data (Defraigne et al. 1994, 1995; Rosat \& Lambert 2009), no FICN resonance has ever been detected at a comfortable level (Rosat et al. 2016), bringing only poor constraints on some geophysical parameters: dynamical ellipticities and densities of the inner core and outer core, deformabilities of the core boundaries under fluid pressure (Dehant et al. 1997), constants characterizing the viscomagnetic coupling at the core boundaries (Mathews et al. 2002), viscosity of the inner core, friction at ICB (Greff-Lefftz et al. 2000, 2002) and topographic torques at the interfaces 
Table 1. Diurnal tidal constituents used for the ETERNA tidal analysis and their associated nutation periods. The waves deriving from the degree- 3 potential are in italic. The names, when available, are from Tamura's (1987) tidal potential catalogue.

\begin{tabular}{ccc}
\hline $\begin{array}{c}\text { Frequency } \\
\text { (cpd) }\end{array}$ & Tidal group name & $\begin{array}{c}\text { Associated nutation period } \\
\text { (sidereal days) }\end{array}$ \\
\hline 0.893244 & $\mathrm{Q}_{1}$ & 9.13 \\
0.898101 & $\rho_{1}$ & 9.56 \\
0.929535 & $\mathrm{O}_{1}$ & 13.66 \\
0.935011 & $\tau_{1}$ & 14.76 \\
0.960971 & $\mathrm{~N}_{1}$ & 23.94 \\
0.965680 & $\mathrm{LK}_{1 \mathrm{x}}$ & 26.98 \\
0.965827 & $\mathrm{LK}_{1}$ & 27.09 \\
0.966137 & $M_{1}$ & 27.32 \\
0.966446 & $\mathrm{NO}_{1}$ & 27.55 \\
0.966593 & $\mathrm{NO}_{1 \mathrm{x}}$ & 27.67 \\
0.971303 & $\chi_{1}$ & 31.81 \\
0.971451 & & 31.96 \\
0.994525 & $\pi_{1}$ & 121.76 \\
0.997115 & $\mathrm{P}_{1 \mathrm{x}}$ & 177.86 \\
0.997262 & $\mathrm{P}_{1}$ & 182.62 \\
0.99788 & & 205.85 \\
1.000000 & $\mathrm{~S}_{1}$ & 365.27 \\
1.001972 & 553 & 1305.82 \\
1.002591 & $\mathrm{~K}_{1 \mathrm{x}-}$ & 6796.6 \\
1.002738 & $\mathrm{~K}_{1}$ & Precession \\
1.002885 & $\mathrm{~K}_{1 \mathrm{x}+}$ & -6796.6 \\
1.005476 & $\Psi_{1}$ & -365.20 \\
1.008214 & $\Phi_{1}$ & -182.61 \\
1.034173 & $\theta_{1}$ & -31.81 \\
1.039030 & $\mathrm{~J}_{1}$ & -27.55 \\
1.039177 & $3 \mathrm{MO}_{1}$ & -27.44 \\
1.070465 & $\mathrm{SO}_{1}$ & -14.76 \\
1.075941 & $\mathrm{OO}_{1}$ & -13.66 \\
1.112232 & $v_{1}$ & -9.13 \\
\hline & & \\
& &
\end{tabular}

(Hinderer et al. 1990; Dehant et al. 1993; Greff-Lefftz \& Legros 1995).

The inversion of the full set of geophysical parameters from the observed VLBI nutations was performed by Mathews et al. (2002) using least-squares. Their model, referred to as MHB, was adopted by the International Astronomy Union (IAU) to represent the Earth's response to tidal forcing (Petit \& Luzum 2010). Later, Koot et al. $(2008,2010)$ re-estimated the values of some of these parameters using a Bayesian inversion of the VLBI nutation in time domain. Since the MHB work, one has $15 \mathrm{yr}$ more of VLBI observations with an increased accuracy. Moreover, one takes benefit of a new celestial reference frame of increased stability (Fey et al. 2015) and some new insights into the influence of the data processing on VLBI products (Gattano et al. 2016). Besides, the basic geophysical parameters inverted in Mathews et al. (2002) and in Koot et al. (2008) are strongly correlated, and some of them were fixed to theoretical values implying strong a priori constraints. Here we directly inverse for the five complex parameters of the FCN and FICN resonance with weak a priori constraint and using these new VLBI products.

In parallel, the length of the surface gravity time-series recorded by superconducting gravimeters (SGs) enables now to perform higher resolution tidal analyses. The resonance effects of the FCN and FICN perturb the amplitudes of diurnal tidal waves. Hence the resonance with the FCN that greatly amplifies the $\Psi_{1}$ wave (corresponding to the retrograde annual nutation) was widely studied and analysed (for instance Neuberg et al. 1987; Defraigne et al. 1994, 1995; Ducarme et al. 2009; Rosat et al. 2009). The presence of
Table 2. Superconducting gravimeter records used in this study.

\begin{tabular}{llcc}
\hline \multicolumn{2}{l}{ Station and location } & Span & Duration in days \\
\hline BE & Brussels, Belgium & $1982-2000$ & 6722 \\
BFO & Black Forest Obs., Germany & $2009-2015$ & 1425 \\
BH & Bad-Homburg, Germany & $2001-2015$ & 5128 \\
CA & Cantley, Canada & $1989-2013$ & 7353 \\
CB & Canberra, Australia & $1997-2013$ & 6028 \\
KA & Kamioka, Japan & $2004-2013$ & 3203 \\
MB & Membach, Belgium & $1995-2014$ & 6767 \\
MC & Medicina, Italy & $1998-2015$ & 5415 \\
ME & Metsahovi, Finland & $1994-2015$ & 7363 \\
MO & Moxa, Germany & $2000-2013$ & 5110 \\
PE & Pecny, Czech Republic & $2007-2015$ & 2921 \\
ST & Strasbourg, France & $1996-2014$ & 6720 \\
SU & Sutherland, South Africa & $2000-2014$ & 4833 \\
VI & Vienna, Austria & $1997-2006$ & 3470 \\
WE & Wettzell, Germany & $1996-2015$ & 6448 \\
\hline
\end{tabular}

the inner core also perturbs some tidal waves, particularly the diurnal tides between $S_{1}$ (prograde annual) and $K_{1}$ (precession). The inversion of the resonances associated with the FCN and FICN in gravity data would constrain additional geophysical parameters that are the Love numbers characterizing the deformability of the core interfaces.

This paper aims at proposing new estimates of the geophysical parameters by performing a Bayesian inversion of the resonance effects due to the FCN and FICN in nutation and in surface gravity data. Even though the resonance effect associated with the FICN has not yet been clearly observed neither in gravity nor in nutation data, the presence of the inner core induces some perturbations on the long-period nutation and on small diurnal tidal constituents. We first recall the resonance models used for the inversion, then we describe the processing of gravity and nutation data. Finally, we perform the Bayesian inversion in frequency domain and discuss the results.

\section{RESONANCE MODELS}

In the following, we note $\sigma$ the frequency in cycle per sidereal day as seen from the rotating Earth and $\sigma^{\prime}=1+\sigma$ the same frequency expressed in space. The frequency domain response of the space motion of the Earth's figure axis to the tidal potential can be written with a transfer function $T$ that expresses the ratio of non-rigid to rigid nutation amplitudes (see e.g. Mathews et al. 1995, 2002). Neglecting the ICW, one has

$T(\sigma)=\frac{e-\sigma}{e+1}\left[1+\sigma^{\prime}\left(\frac{N_{1}}{\sigma-s_{1}}+\frac{N_{2}}{\sigma^{\prime}-s_{2}}+\frac{N_{3}}{\sigma^{\prime}-s_{3}}\right)\right]$,

where $e$ is the Earth flattening. The bracketed terms express the CW, FCN and FICN resonances, respectively, with

$s_{1}=\frac{A}{A_{\mathrm{m}}}(e-\kappa)=-e N_{1}$,

$s_{2}=-\frac{A}{A_{\mathrm{m}}}\left(e_{\mathrm{f}}-\beta+K_{\mathrm{CMB}}+\frac{A_{\mathrm{s}}}{A_{\mathrm{f}}} K_{\mathrm{ICB}}\right)$,

$s_{3}=\frac{A}{A_{\mathrm{m}}}\left(\alpha_{2} e_{\mathrm{s}}+v-K_{\mathrm{ICB}}\right)$,

$N_{2}=\frac{A_{\mathrm{f}}}{A_{\mathrm{m}}}(e-\gamma)$,

where $A, A_{\mathrm{m}}, A_{\mathrm{f}}$ and $A_{\mathrm{s}}$ are the mean equatorial moments of inertia of the Earth, the mantle, the fluid outer core, and the solid inner 

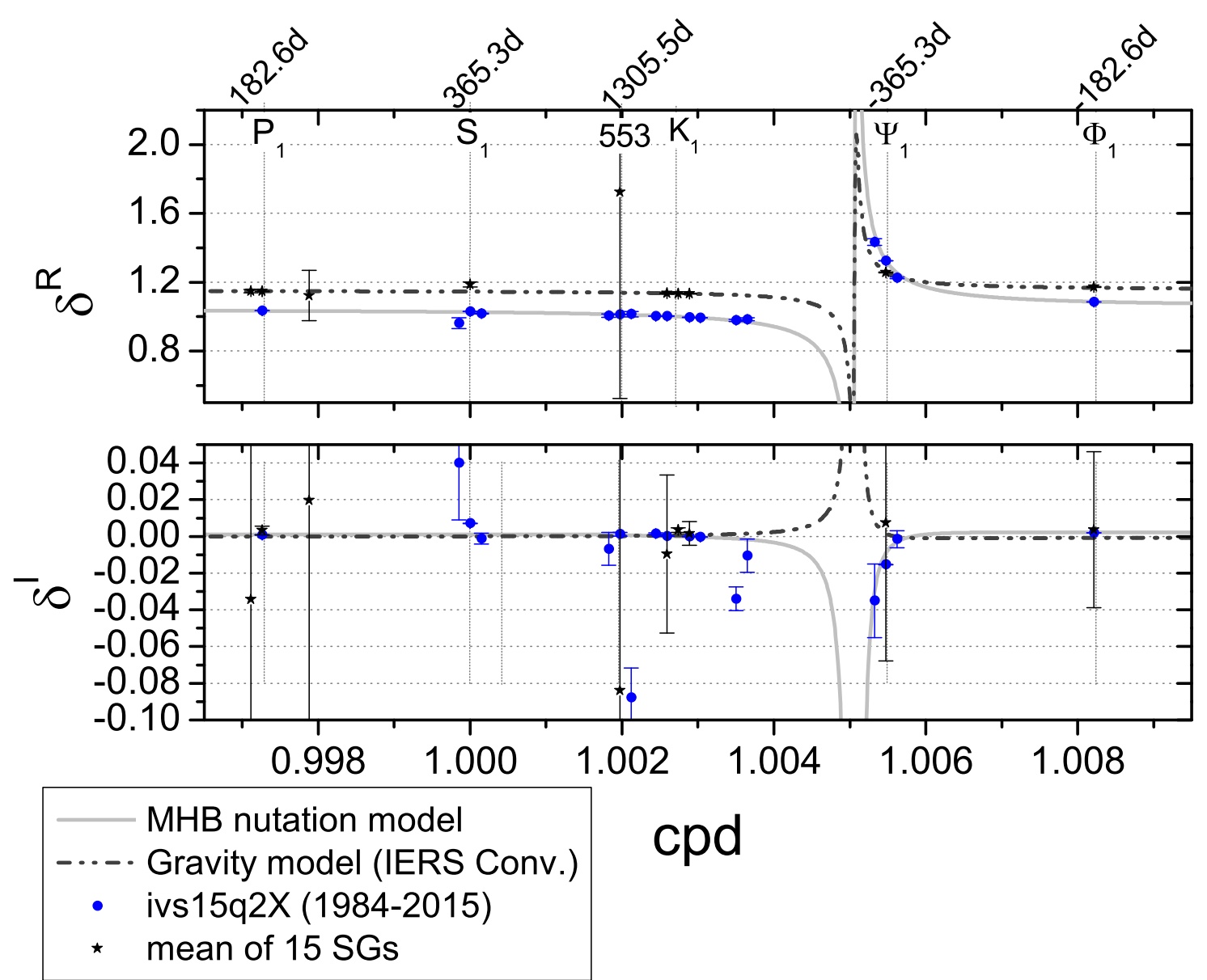

Figure 1. Real and imaginary parts of the observed transfer functions for gravity (stars) and nutation (dots) obtained respectively from the weighted mean of 15 SGs and from VLBI measurements (1984-2015.5). The MHB model for nutation is plotted with a thick grey line and the one for gravity with a dashed black line. In vertical grey lines, we have indicated the frequency (in cycle per solar day) in a terrestrial reference frame of the diurnal tides as well as their respective nutations (in sidereal days) in a celestial reference frame. The frequency axis is given in cycle per day in a terrestrial reference frame.

core, respectively. The flattenings $e_{\mathrm{f}}$ and $e_{\mathrm{s}}$ are relevant to the fluid and solid inner cores. The compliance $\kappa=e k / k_{\mathrm{s}}$, where $k$ and $k_{\mathrm{s}}$ are the elastic and fluid Love number, respectively, expresses the deformability at the surface under degree 2 tidal forcing. The definition of other quantities is given in Mathews et al. (2002). The full expression for $N_{3}$ can be found in, for example, Dehant et al. (2005).

The tidal variations observed at the Earth's surface are induced by the direct effects of the tidal potential on the Earth and the indirect effects on deformations and mass redistribution. The direct, deformation, and mass redistribution effects of the centripetal potential due to the Earth's wobble must also be considered. Besides, the inertial pressure at the core-mantle boundary $(\mathrm{CMB})$ due to the differential rotation between the mantle and the core induces a deformation of the CMB as well as mass redistribution in the mantle that generate also time variations of the gravity field. Summing all these effects and dividing by the gravity variations for a non-rotating rigid Earth lead to the tidal gravimetric factor (Neuberg et al. 1987; Hinderer et al. 1991; Legros et al. 1993):

$$
\begin{aligned}
T_{\mathrm{g}}(\sigma) & =\delta_{2}(1-e)-\frac{e N_{2}}{\sigma^{\prime}-s_{2}}\left[\delta_{2} \sigma^{\prime}+\bar{\delta}_{1} \frac{A}{A_{\mathrm{f}}}\right]+\frac{N_{3, \mathrm{~g}}}{\sigma^{\prime}-s_{3}}, \\
& =\delta_{\text {ref }}+\frac{N_{2, \mathrm{~g}}}{\sigma^{\prime}-s_{2}}+\frac{N_{3, \mathrm{~g}}}{\sigma^{\prime}-s_{3}},
\end{aligned}
$$

where the resonance strengths $N_{2, \mathrm{~g}}$ and $N_{3, \mathrm{~g}}$ have different expressions than for the nutations (Legros et al. 1993). Following Florsch \& Hinderer (2000) and Rosat et al. (2009), the value of $\delta_{\text {ref }}$ will be taken equal to the mean value of the observed gravimetric factors for the lowest and highest frequency diurnal constituent, that is to say $Q_{1}$ and $v_{1}$ in our case (Table 1). The influence of this value on the retrieved FCN parameters appears to be negligible (Rosat et al. 2009).

\section{DATA PREPARATION}

We used nutation measurements provided by the International VLBI Service for Geodesy and Astrometry combination ivs15q2X (IVS, Böckmann et al. 2007; Schuh \& Behrend 2012) for 3770 selected VLBI sessions since 1984. Prograde and retrograde amplitudes of the terms listed in table 1 of the MHB paper and linear trends were obtained by weighted least-squares. The fit was done iteratively and associated with the determination of an error floor $f$ and an error scale factor $s$ aiming at inflating the error $\epsilon$ given in the IVS file following $(s \epsilon)^{2}+f^{2}$. With recalibrated errors, the fit of nutation amplitudes achieves both a minimum sum of squared differences between observations and model and a standard deviation of the residuals consistent with the errors (Herring et al. 1991, 2002; Gattano et al. 2016). We found $f=0.96$ and $s=0.094$ mas. The obtained nutation amplitudes were corrected for effects that are 
not or nonlinearly linked to non-rigidity, including the geodetic nutation, the $S_{1}$ atmospheric tide, and the contribution of secondorder terms in the dynamical equations (table 7 of the MHB paper). Finally we formed the ratios of the observed amplitudes to their rigid counterparts given by REN 2000 (Souchay et al. 1999).

The gravimetric records were selected in terms of length of records from the worldwide network of SGs (Crossley et al. 1999; see Table 2). These SG time-varying gravity records have been preprocessed to remove any gaps, spikes, steps and other disturbances so that a tidal analysis with the ETERNA 3.4 software package (Wenzel 1996) is possible. The minute data were resampled to $1 \mathrm{hr}$ (using a filter with a cut-off period of $3 \mathrm{hr}$ ). ETERNA performs a least-square fit to tides, local air pressure and instrumental drift to give complex gravimetric factors, residual gravity, an adjusted barometric admittance, and a polynomial drift function. ETERNA analysis is performed on tidal groups. The number of tidal waves that can be determined and the precision obtained in our tidal analyses depend on the record length and on the noise characteristics of the instrument used. We choose the 28 main tidal constituents in the diurnal frequency band given by the tidal potential catalogue of Hartmann \& Wenzel (1995) between $\mathrm{Q}_{1}$ and $v_{1}$ (Table 1). Note that the degree-3 $M_{1}$ diurnal wave was analysed with ETERNA but was not used for the inversion since only degree- 2 tides are involved in the resonance model. The complex gravimetric factors are then corrected for the ocean tide loading effect according to the EOT11a ocean model (Savcenko \& Bosch 2011). The computation was done using the ocean tide loading provider website of M. S. Bos and H.-G. Scherneck (http://holt.oso.chalmers.se/loading). From the 28 complex gravimetric factors at each SG site, we computed the weighted averages (Fig. 1) based on the uncertainties given by ETERNA software.

\section{ANALYSIS AND RESULTS}

Mathews et al. (2002) and Koot et al. (2008) estimated the following basic Earth parameters (BEP): $\kappa, \gamma, e_{\mathrm{f}}+K_{\mathrm{CMB}}, \beta, K_{\mathrm{ICB}}, \alpha_{2} e_{\mathrm{s}}+v$ and $N_{3}$. These parameters are all complex numbers, but following Mathews et al. (2002) we will ignore imaginary parts of $\kappa, \gamma, \beta$ and $v$ since they are very small.

In the following, the superscripts $\mathrm{R}$ and I stand for the real and imaginary parts of the complex parameters, respectively. Correlation matrix of BEP for nutations (Fig. 2) reveals that $e_{\mathrm{f}}+K_{\mathrm{CMB}}^{R}$, $\beta$ and $\kappa$ are 100 per cent correlated (or anti-correlated). The value of $\alpha_{2} e_{\mathrm{s}}+v$ was fixed to the theoretical PREM value of Mathews et al. (1991) in Koot et al. (2008), which automatically leads to a constrained value for the FICN frequency. Besides $K_{\text {ICB }}^{R}$ is also correlated to $\beta$ and $\kappa$, and $\beta$ was fixed to its theoretical PREM value too. The pairs $\left(\kappa, N_{3}^{R}\right)$ and $\left(\beta, N_{3}^{R}\right)$ are correlated at about 50 per cent, while $N_{3}^{R}$ and $\gamma$ at about 60 percent. There is also a strong trade-off between the real (resp. imaginary) parts of $K_{\mathrm{CMB}}$ and $K_{\mathrm{ICB}}$ and between $N_{2}$ and $N_{3}$. It is hence difficult to inverse for all seven complex BEP without strong a priori constraints. As a consequence, we chose to inverse for five parameters consisting of $N_{1}\left(=-s_{1} / e\right), N_{2}, N_{3}, s_{2}$ and $s_{3}$ (Fig. 2b). Correlations have globally decreased although $N_{2}$ and $N_{3}$ are still strongly correlated to about 70 percent. A correlation also shows up between $s_{2}$ and $N_{2}$. For gravity data, we inverse for the two resonance strengths $N_{2, g}$ and $N_{3, g}$ and the two complex frequencies $s_{2}$ and $s_{3}$.

We already applied in the past a Bayesian inversion to estimate the period and quality factor of the FCN from gravimetric data (Rosat et al. 2009) and from both VLBI nutation and SG data
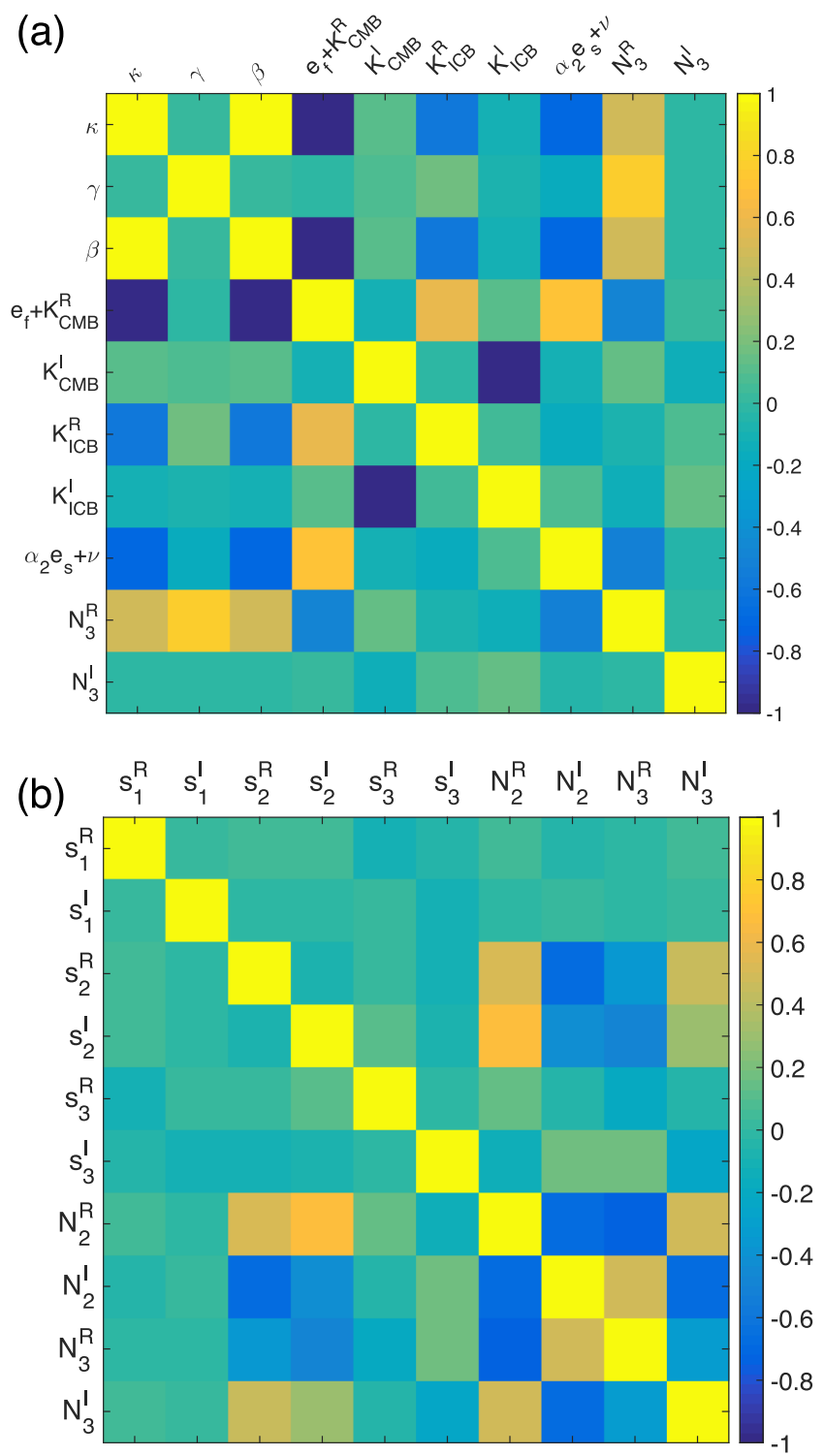

Figure 2. Correlation matrices for two sets of parameters: (a) for the seven Basic Earth Parameters defined in Mathews et al. (2002; the imaginary parts of four of them are ignored); (b) for the five complex parameters defined in the resonance model for the Chandler Wobble $(\mathrm{CW})$, the Free Core Nutation (FCN) and the Free Inner Core Nutation (FICN). The $R$ and $I$ exponents respectively mean real and imaginary parts of the parameter.

(Rosat \& Lambert 2009). Here, since the number of parameters involved in the inversion process is large, we cannot compute the full probability density functions and we need a sampling algorithm. We use the Metropolis-Hastings algorithm (Metropolis et al. 1953; Hastings 1970) with a Markov-chain Monte-Carlo (MCMC) method (e.g. Aster et al. 2013) to perform the Bayesian inversion of the resonance parameters. MCMC methods are easily applied to both linear and nonlinear problems, since they depend only on the forward model and associated likelihood computations. A Markov chain is a sequence of random variables, where the probability value depends solely on the previous value. The Metropolis-Hastings sampler is an algorithm that generates a Markov chain with a specified limiting distribution. From a posterior distribution, this algorithm will produce samples that characterize the posterior distribution of an inverse problem. The implementation of the MetropolisHastings sampler is subjected to a likelihood-based test for which we 

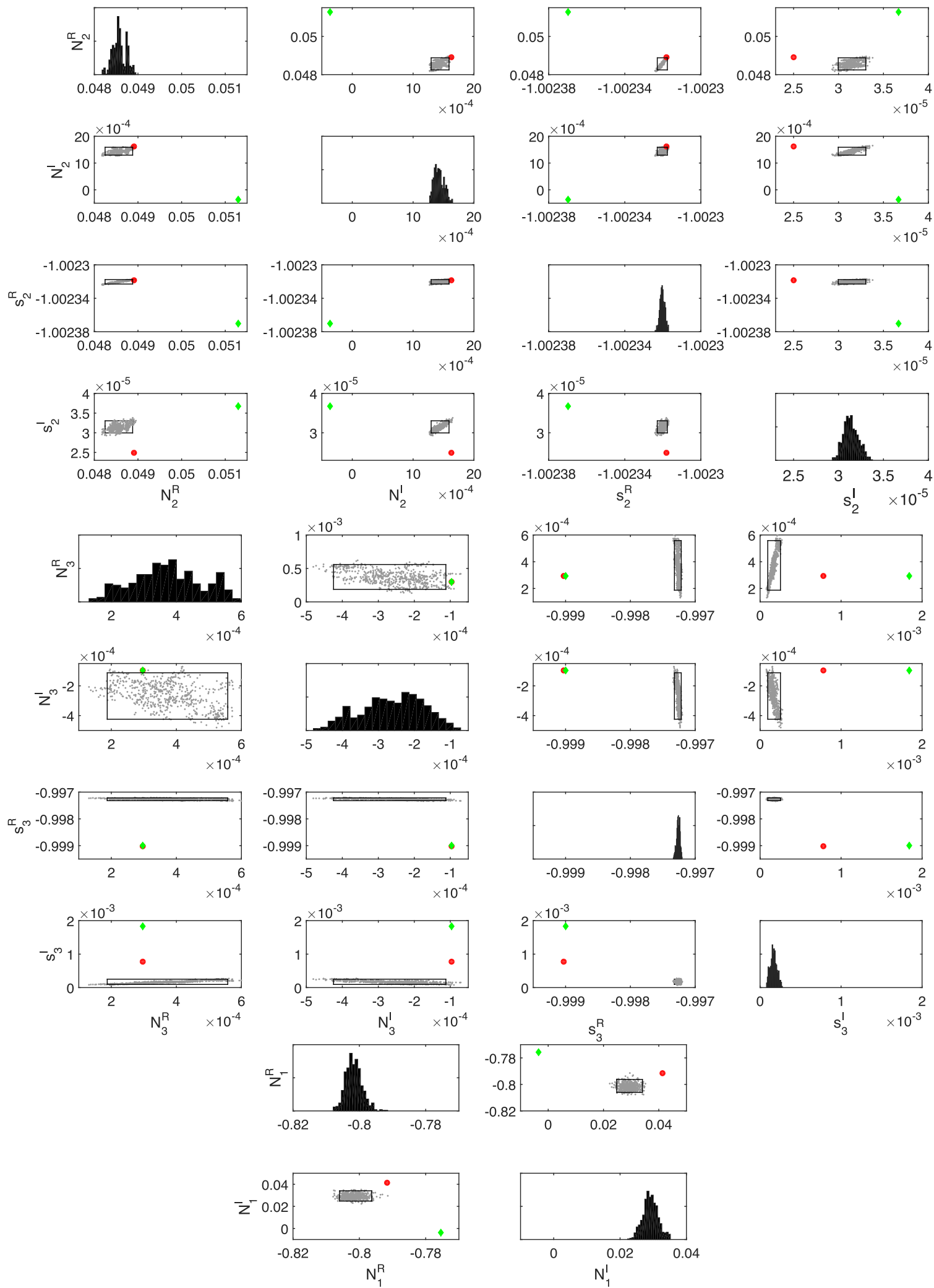

Figure 3. Sampled posterior distributions for simultaneous estimation of $s_{2}, N_{2}, s_{3}, N_{3}$ and $N_{1}$ obtained from nutation. The MHB values are plotted with a red dot and the values obtained by Koot et al. (2008) are represented by a green diamond. The boxes represent the 95 per cent probability intervals estimated from the Markov-chain Monte-Carlo posterior distribution. 

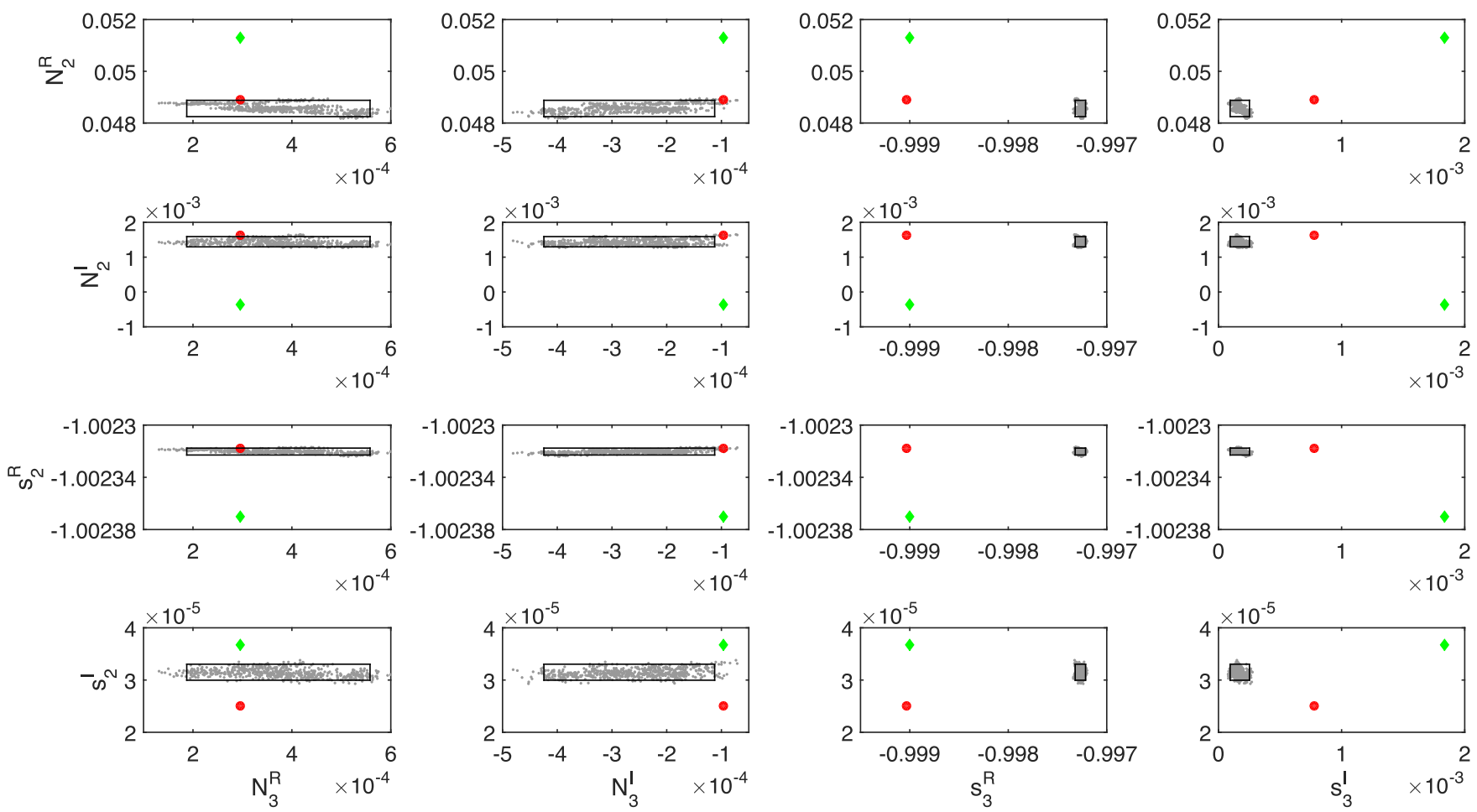

Figure 4. Sampled posterior distributions for simultaneous estimation of $s_{2}, s_{3}, N_{2}$ and $N_{3}$ obtained from nutation. The MHB values are plotted with a red dot and the values obtained by Koot et al. (2008) are represented by a green diamond. The boxes represent the 95 per cent probability intervals estimated from the Markov-chain Monte-Carlo posterior distribution.

introduce an acceptance ratio. Some studies suggest that the algorithm is optimally tuned when the acceptance ratio of the new model is between about 20 per cent and 50 per cent (e.g. Gelman et al. 2003; Aster et al. 2013). We use this ratio of number of accepted models over the number of posterior distribution samples as an acceptance test for our final model. In order to sample the posterior model space, we use a multivariate normal generator with zero covariances and variances defined by the step sizes used to generate the candidate models. Smaller steps will result in higher acceptance ratios, but the algorithm may be unacceptably slow. Conversely, larger steps will result in lower acceptance ratios (Aster et al. 2013).
We assume uniform prior distributions for the parameters that we inverse (the ones of Fig. 2(b)) since we do not know a priori their distributions (e.g. Florsch \& Hinderer 2000). Prior information, particularly in ill-posed inverse problem, is of critical importance because it is conditioning the posterior solution. In other words, a completely unresolved parameter has a posterior marginal density function that follows the a priori one. So the more the a posteriori density function differs from the a priori one, the more the parameter has been resolved (Tarantola \& Valette 1982). The bounds are chosen so that the MHB values as well as the theoretical predictions for different Earth models (e.g. Mathews et al.

Table 3. 95 per cent CI for the estimated resonance parameters and comparison with the results of Koot et al. (2008) and Mathews et al. (2002).

\begin{tabular}{lcccc}
\hline Parameter & Nutation & Gravity & Koot et al. (2008) & Mathews et al. (2002) \\
\hline$N_{2}^{\mathrm{R}}$ & $(0.0482,0.0488)$ & $(4.5,7.3) \times 10^{-4}$ & 0.05129 & 0.0489108 \\
$N_{2}^{\mathrm{I}}$ & $(1.23,1.53) \times 10^{-3}$ & $(-1.7,0.7) \times 10^{-4}$ & $-0.362 \times 10^{-3}$ & $1.62916 \times 10^{-3}$ \\
$T_{\mathrm{FCN}}$ & $(-430.1,-429.3)$ & $(-515,-396)$ & -421.94 & $(-429.93,-430.48)$ \\
$Q_{\mathrm{FCN}}$ & $(15392,16866)$ & $(7763,320888)$ & 13665 & 20000 \\
$N_{3}^{\mathrm{R}}$ & $(1.6,8.4) \times 10^{-4}$ & $(-7.2,9.1) \times 10^{-4}$ & $2.9584 \times 10^{-4}$ & $2.95844 \times 10^{-4}$ \\
$N_{3}^{\mathrm{I}}$ & $(-2.0,3.6) \times 10^{-4}$ & $(-7.4,9.4) \times 10^{-4}$ & $-9.577 \times 10^{-5}$ & $-9.57707 \times 10^{-5}$ \\
$T_{\mathrm{FICN}}$ & $(362,414)$ & $(28,725)$ & 1000 & $(930,1140)$ \\
$Q_{\mathrm{FICN}}$ & $(2600,6807)$ & $(75,3083)$ & 271 & 677 \\
$N_{1}^{\mathrm{R}}$ & $(-0.804,-0.796)$ & - & -0.7755 & -0.791653 \\
$N_{1}^{\mathrm{I}}$ & $(0.027,0.037)$ & - & -0.00358 & 0.0414503 \\
$T_{\mathrm{CW}}$ & $(425,430)$ & - & 393 & 430.3 \\
$Q_{\mathrm{CW}}$ & $(19,40)$ & - & 108 & 88.4 \\
\hline
\end{tabular}



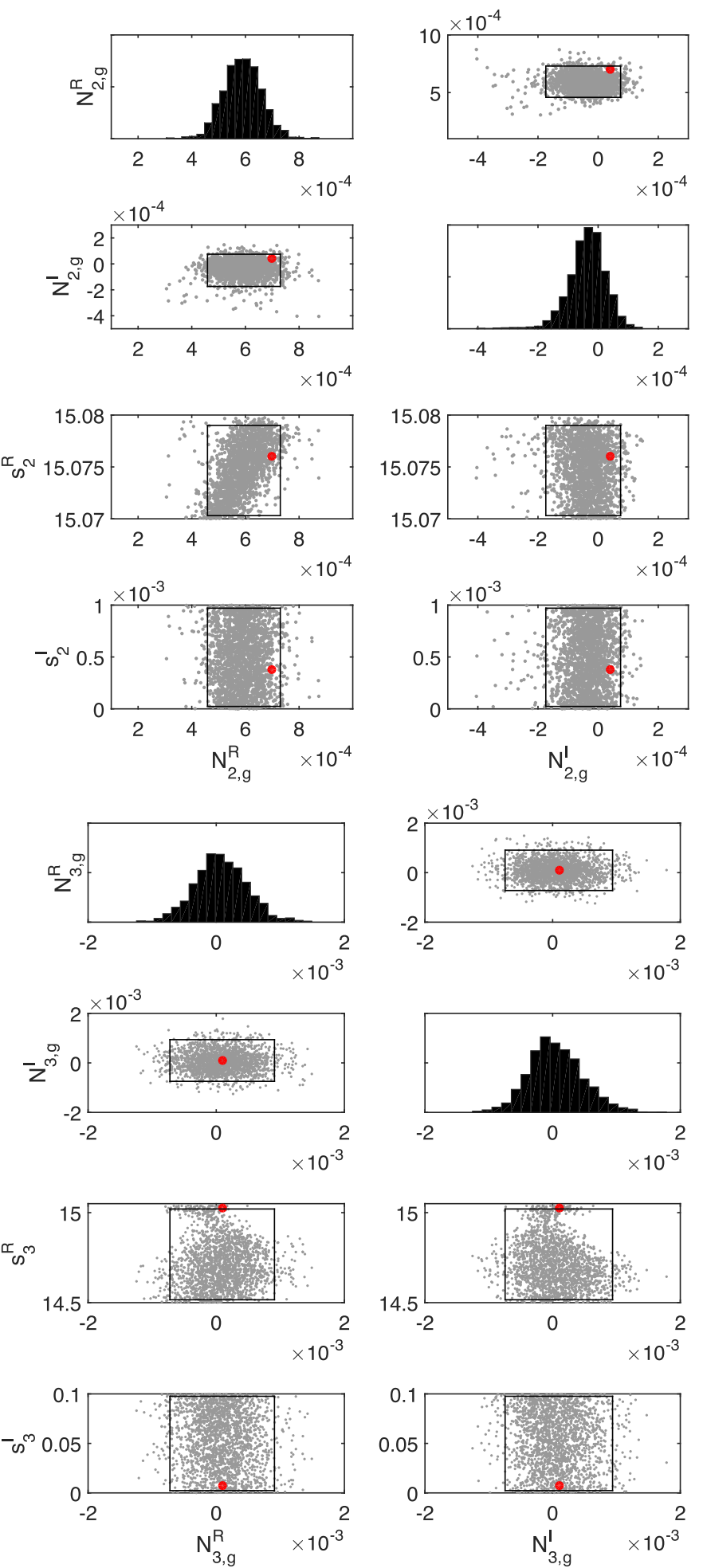
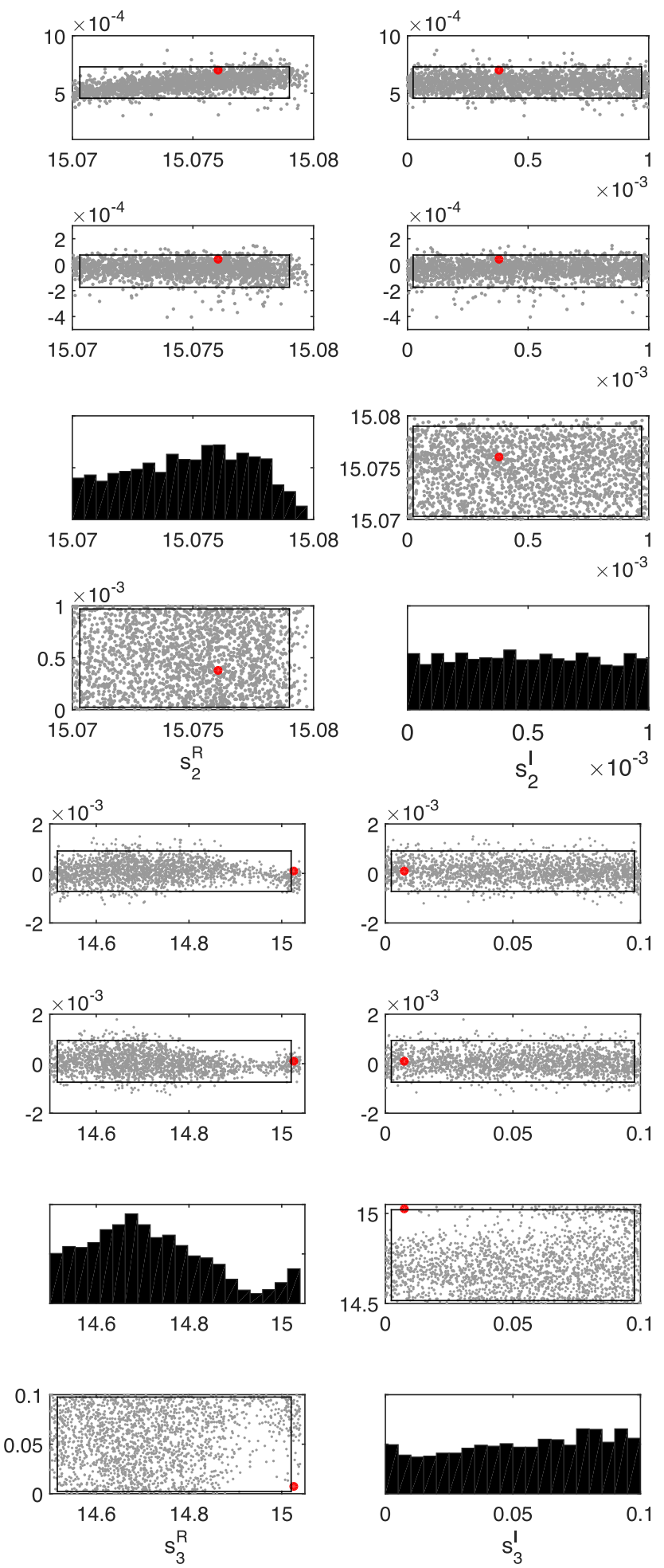

Figure 5. Sampled posterior distributions for the eight parameters obtained from gravimetric tidal analyses. The MHB values are plotted with a red dot. The boxes represent the 95 per cent probability intervals estimated from the Markov-chain Monte-Carlo posterior distribution.

1991, 1995) are within the ranges. Contrary to Koot et al. (2008), we did not impose any a priori Gaussian distribution of the modelling errors. We rather preferred to use the corrections of Herring et al. (2002) to the standard deviations of VLBI nutation data as mentioned in Section 3. In such a way, no Gaussian a priori is introduced.
The sampled posterior distributions of the geophysical parameters obtained from nutations are plotted in Figs 3 and 4. Indeed we sample the posterior distributions using 600000 steps, but only 600 retrieved samples are shown (down-sampling by keeping every 1000th calculated sample) to produce samples with a low correlation (Aster et al. 2013). These probability distributions do not 
provide a single model but a range of possible solutions. When we want to single out a representative model, it is convenient to select the one corresponding to the largest probability (Aster et al. 2013). This model is called the Maximum A Posteriori (MAP) model. Another solution is to select the mean value of the posterior distribution. When the distribution is normal, the MAP and posterior mean models are identical. However, when the probability density functions are not unimodal, using the MAP or the mean model may be dangerous in the sense that the obtained model from these estimators may be out of the confidence intervals. That is why we show only the plots of the sampled posterior probability density functions which contain more information on the estimated parameters.

The 95 per cent confidence intervals (CI) for the parameter values are given in Table 3. The FCN, FICN and $\mathrm{CW}$ periods and quality factors are respectively noted $T_{\mathrm{FCN}}, T_{\mathrm{FICN}}, T_{\mathrm{CW}}, Q_{\mathrm{FCN}}, Q_{\mathrm{FICN}}$ and $Q_{\mathrm{CW}}$. For comparison, we also give the values of Koot et al. (2008) and Mathews et al. (2002). Tilted shapes of the joint distributions are the signature of correlations. The 95 per cent CI for the FCN and for the $\mathrm{CW}$ are in good agreement with MHB but less with Koot et al. (2008). For the FICN, the most probable solutions do not agree with the previous estimates by Koot et al. (2008) or Mathews et al. (2002). The 95 per cent CI propose a solution around the annual nutation (or $S_{1}$ tide) instead of $\sim 1000$ d (Mathews et al. 2002; Koot et al. 2008), hence in closer agreement with the theoretically predicted values of, for example, Dehant et al. (1993), Greff-Lefftz et al. (2000) or Rogister (2001) for a PREM (Dziewonski \& Anderson 1981) Earth model. A consequence of the correlations between parameters is the error on the FCN parameters that is larger than in previous studies (for instance Rosat \& Lambert 2009) where the FICN was not considered.

We see from Fig. 3 that the FICN is poorly, if at all, constrained by the VLBI nutation data. The 95 per cent CI lead to a FICN period between 362 and 414 days and a quality factor between 2600 and 6807 (see Table 3). We suspect that the inversion adjusts a resonance model to the annual nutation ( $S_{1}$ tide) term, or more specifically to the remaining annual term since the later was corrected in the processing of VLBI data. Indeed, when we try the MCMC inversion without the annual nutation, the posterior distribution shows that its period could be any value between the prior bounds with a 95 per cent CI, that is, between 327 and 9782 days, and a Q-value between 251 and 1024. So this clearly shows that VLBI data cannot constrain the FICN frequency. For a test, we have also applied the classical Bayesian inversion as used in Rosat et al. (2009) and Rosat \& Lambert (2009) to the FICN parameters alone, by fixing the CW and FCN parameters to MHB values. The obtained joint density probability functions confirm the results obtained with the MCMC method on the ten parameters: the FICN period could take any value in the prograde band between our a priori bounds. This is not surprising since the resonance is not observed and the effect of the inner core on the long period nutation is much smaller than the errors on the estimated nutation amplitudes. Please note that similarly to Mathews et al. (2002), $s_{1}$ is indeed the resonance frequency of the $\mathrm{CW}$ excited by the retrograde $18.6 \mathrm{yr}$ nutation. The resonance frequency is not equal to the frequency of the free mode when the resonance model has frequency dependent parameters, which is the case of the transfer function in eq. (1). In order to obtain the frequency of the free mode $\sigma_{\mathrm{CW}}$ from the resonance frequency $s_{1}$, we use the same equation (D5) as in Mathews et al. (2002), that is,

$\sigma_{\mathrm{CW}}=s_{1}+\frac{A}{A_{m}}\left[\Delta \kappa\left(\sigma_{18.6}\right)-\Delta \kappa\left(\sigma_{\mathrm{CW}}\right)\right]$, where $\Delta \kappa\left(\sigma_{18.6}\right)=(-5.646+12.020 i) \times 10^{-5}$ is the combined increment from anelasticity and ocean tide at the frequency of the retrograde 18.6 yr nutation, and $\Delta \kappa\left(\sigma_{\mathrm{CW}}\right)=(19.481-1.205 i) \times 10^{-5}$ the one at the Chandler frequency (Mathews et al. 2002).

We now try to inverse for the FCN and FICN parameters from the surface gravity observations. The sampled posterior distributions for the FCN and FICN parameters are plotted in Fig. 5. We do not show here the joint distributions since they do not bring any valuable information. The results for the FCN are in good agreement with the results obtained from VLBI. The possible solutions for the FICN have periods between 28 and $725 \mathrm{~d}$ within the 95 per cent CI. If we take an upper limit for the prior on the FICN frequency larger than the $K_{1}$ frequency, that is to say, if we accept a priori retrograde periods, we obtain a certain amount of samples of the posterior distribution close to the values of Mathews et al. (2002) and Koot et al. (2008), that is, close to 1000 days. This is due to the influence of the FCN resonance.

\section{CONCLUSIONS}

We analysed a longer VLBI nutation solution with a different strategy for processing data as was previously done in Mathews et al. (2002) and Koot et al. (2008). In contrast to these previous studies, we have decided not to incorporate subjective judgments into the model, that is why we chose uniform prior distributions with broad limits and with a limited number of parameters. We have shown that both the VLBI nutation and the gravimeter data suggest values of the FICN frequency lower than previous estimates. There is no unique solution for the FICN frequency, but rather a range of possible values. The posterior distributions for the FICN frequency clearly demonstrate the advantage of a Bayesian scheme over a least-squares inversion method since the posterior density distributions are clearly not Gaussian, except for the resonance strengths $N_{1}, N_{2}$ and $N_{3}$ which approximate the normality. Gravimetric data also constrain the FICN period, if the observed resonance is actually associated with the FICN, but towards lower values close to the annual nutation $\left(S_{1}\right.$ tide). When we reject the annual nutation term, the posterior samples indicate that any FICN frequency could fit the data with a 95 per cent confidence interval.

It emerges from these results that both gravity and nutation data are not precise enough to put objective constraints on the FICN frequency and damping factor. The existing correlations between the basic Earth parameters prevent from robust and independent estimates of the coupling constants and of the compliances at the core boundaries from the data. As long as the resonance associated with the FICN is not clearly identified in the data, it will be difficult to estimate additional geophysical parameters characterizing the coupling and deformation at the core boundaries without strong a priori constraints. Longer time-series are still needed to further improve the determination of the small diurnal tidal constituents and of the associated long-period nutations which are most affected by the FICN rotational mode.

\section{ACKNOWLEDGEMENTS}

We gratefully acknowledge the Groupe de Recherche de Géodésie Spatiale (GRGS) and the CNRS-INSU Programme National de Planétologie for their financial support. We thank also the managers of SG instruments for sharing their data within the GGP (data downloaded from http://isdc.gfz-potsdam.de) and to the IVS for 
their constant effort in scheduling and correlating VLBI sessions. Finally, we thank Nicolas Florsch and an anonymous referee for their reviews.

\section{REFERENCES}

Amoruso, A., Botta, V. \& Crescentini, L., 2012. Free Core Resonance parameters from strain data: sensitivity analysis and results from the Gran Sasso (Italy) extensometers, Geophys. J. Int., 189, 923-936.

Aster, R., Borchers, B. \& Thurber, C., 2013. Parameter Estimation and Inverse Problems, 2nd edn, Elsevier Academic Press, 360 pp.

Blum, P.A., Hatzfeld, D. \& Wittlinger, G., 1973. Résultats expérimentaux sur la fréquence de résonance dueà l'effet dynamique du noyau liquide, C. R. Acad. Sci. Paris B, 277, 241-244.

Böckmann, S., Artz, T., Nothnagel, A. \& Tesmer, V., 2007. Comparison and combination of consistent VLBI solutions, in Proceedings of the 18th European VLBI for geodesy and astrometry working meeting, vol. 79, pp. 82-87, eds Böhm, J., Pany, A. \& Schuh, H., Vienna University of Technology.

Crossley, D. et al., 1999. Network of superconducting gravimeters benefits a number of disciplines, EOS, Trans. Am. geophys. Un., 80(11), $121-126$.

Defraigne, P., Dehant, V. \& Hinderer, J., 1994. Stacking gravity tide measurements and nutation observations in order to determine the complex eigenfrequency of the nearly diurnal free wobble, J. geophys. Res., 99, 9203-9213.

Defraigne, P., Dehant, V. \& Hinderer, J., 1995. Correction to “Stacking gravity tide measurements and nutation observations in order to determine the complex eigenfrequency of the nearly diurnal free wobble", J. geophys. Res., 100(B2), 2041-2042.

Dehant, V., Hinderer, J., Legros, H. \& Lefftz, M., 1993. Analytical approach to the computation of the earth, the outer core and the inner core rotational motions, Phys. Earth planet. Inter., 76, 259-282.

Dehant, V., Feissel, M., Defraigne, P., Roosbeek, F. \& Souchay, J., 1997. Could the energy near the FCN and the FICN be explained by luni-solar or atmospheric forcing?, Geophys. J. Int., 130, 535-546.

Dehant, V., de Viron, O. \& Greff-Lefftz, M., 2005. Atmospheric and oceanic excitation of the rotation of a three-layer Earth, Astron. Astrophys., 438, 1149-1161.

de Vries, D. \& Wahr, J.M., 1991. The effects of the solid inner core and nonhydrostatic structure on the Earth's forced nutations and Earth tides, J. geophys. Res. B, 96, 8275-8293.

Ducarme, B., Rosat, S., Vandercoilden, L., Jian-Qiao, X. \& Heping, S., 2009. European tidal gravity observations: comparison with earth tide models and estimation of the Free Core Nutation (FCN) parameters, in Observing our Changing Earth, pp. 523-532, ed. Sideris, M.G., Springer.

Ducarme, B., Sun, H.-P. \& Xu, J.-Q., 2007. Determination of the free core nutation period from tidal gravity observations of the GGP superconducting gravimeter network, J. Geod., 81, 179-187.

Dumberry, M., 2009. Influence of elastic deformations on the inner core wobble, Geophys. J. Int., 178, 57-64.

Dziewonski, A.M. \& Anderson, D.L., 1981. Preliminary reference Earth model (PREM), Phys. Earth planet. Inter., 25, 297-356.

Florsch, N. \& Hinderer, J., 2000. Bayesian estimation of the free core nutation parameters from the analysis of precise tidal gravity data, Phys. Earth planet Inter., 117, 21-35.

Fey, A.L. et al., 2015. The second realization of the International Celestial Reference Frame by very long baseline interferometry, Astron. J., 150(2), doi:10.1088/0004-6256/150/2/58.

Gattano, C., Lambert, S. \& Bizouard, C., 2016. Observation of the Earth's nutation by the VLBI: how accurate is the geophysical signal, J. Geod., in press, doi:10.1007/s00190-016-0940-7.

Gelman, A., Carlin, J.B., Stern, H.S. \& Rubin, D.B., 2003. Bayesian Data Analysis, 2nd ed., Chapman \& Hall/CRC Press.

Greff-Lefftz, M. \& Legros, H., 1995. Core-mantle coupling and viscoelastic deformations, Phys. Earth planet. Inter., 90, 115-135.
Greff-Lefftz, M., Legros, H. \& Dehant, V., 2000. Influence of the inner core viscosity on the rotational eigenmodes of the Earth, Phys. Earth planet. Inter, 122, 187-204.

Greff-Lefftz, M., Dehant, V. \& Legros, H., 2002. Effects of inner core viscosity on gravity changes and spatial nutations induced by luni-solar tides, Phys. Earth planet. Inter., 129, 31-41.

Hartmann, T. \& Wenzel, H.-G., 1995. The HW95 tidal potential catalogue, Geophys. Res. Lett., 22(24), 3553-3556.

Hastings, W.K., 1970. Monte Carlo sampling methods using Markov chains and their applications, Biometrika, 57, 97-109.

Herring, T.A., Buffet, B.A., Mathews, P.M. \& Shapiro, I.I., 1991. Forced nutations of the Earth: influence of inner core dynamics, 3. Very long interferometry analysis, J. geophys. Res., 91, 4755-4765.

Herring, T.A., Mathews, P.M. \& Buffet, B.A., 2002. Modeling of nutationprecession: very long baseline interferometry results, J. geophys. Res., 107, doi:10.1029/2001JB000165.

Hinderer, J., Legros, H., Jault, D. \& Le Mouël, J., 1990. Core-mantle topography torque: a spherical harmonic approach and implications for the excitation of the Earth's rotation by core motions, Phys. Earth planet. Inter, 59, 329-341.

Hinderer, J., Zürn, W. \& Legros, H., 1991. Interpretation of the strength of the nearly diurnal free wobble resonance from stacked gravity tide observations, in Proc. 11th Int. Symp. Earth Tides, pp. 549-555, ed. Kakkuri, J., Schweitzerbart. Verlag.

IERS Conventions, 2010. Gérard Petit and Brian Luzum (eds.). (IERS Technical Note 36) Frankfurt am Main: Verlag des Bundesamts für Kartographie und Geodäsie, 2010, 179 pp., ISBN 3-89888-989-6.

Koot, L., Rivoldini, A., de Viron, O. \& Dehant, V., 2008. Estimation of Earth interior parameters from a Bayesian inversion of very long baseline interferometry nutation time series, J. geophys. Res., 113, B08414, doi:10.1029/2007JB005409.

Koot, L., Dumberry, M., Rivoldini, A., de Viron, O. \& Dehant, V., 2010. Constraints on the coupling at the core-mantle and inner core boundaries inferred from nutation observations, Geophys. J. Int., 182, 1279-1294.

Lambert, S.B. \& Dehant, V., 2007. The Earth's core parameters as seen by the VLBI, Astron. Astrophys., 469, 777-781.

Legros, H., Hinderer, J., Lefftz, M. \& Dehant, V., 1993. The influence of the solid inner core on gravity changes and spatial nutations induced by luni-solar tides and surface loading, Phys. Earth planet. Inter., 76, 283-315.

Mathews, P.M., Buffett, B.A., Herring, T.A. \& Shapiro, I.I., 1991. Forced nutations of the Earth: influence of inner core dynamics: 2. Numerical results and comparisons, J. geophys. Res., 96, 8243-8257.

Mathews, P.M., Buffet, B.A. \& Shapiro, I.I., 1995. Love numbers for diurnal tides: relation to wobble admittances and resonance expansions, $J$. geophys. Res., 100(B7), 9935-9948.

Mathews, P.M., Herring, T.A. \& Buffet, B.A., 2002. Modeling of nutation-precession: new nutation series for nonrigid Earth and insights into the Earth's interior, J. geophys. Res., 107(B4), ETG 3-1ETG 3-26.

Metropolis, N., Rosenbluth, A.W., Rosenbluth, M.N., Teller, A.H. \& Teller, E., 1953. Equations of state calculations by fast computing machine, $J$. Chem. Phys., 21, 1087-1091.

Neuberg, J. \& Zürn, W., 1986. Investigation of the nearly diurnal resonance using gravity, tilt and strain data simultaneously, in Proc., 10th Int. Symp. Earth Tides, pp. 305-311, ed. Vieira, R., Cons. Sup. Inv. Cient.

Neuberg, J., Hinderer, J. \& Zürn, W., 1987. Stacking gravity tide observations in Central Europe for the retrieval of the complex eigenfrequency of the nearly diurnal free wobble, Geophys. J. R. astr. Soc., 91, $853-868$.

Rogister, Y., 2001. On the diurnal and nearly diurnal free modes of the Earth, Geophys. J. Int., 144, 459-470.

Rosat, S. \& Lambert, S.B., 2009. Free core nutation resonance parameters from VLBI and superconducting gravimeter data, Astron. Astrophys., 503, 287-291.

Rosat, S., Florsch, N., Hinderer, J. \& Llubes, M., 2009. Estimation of the Free Core Nutation parameters from SG data: sensitivity study and 
comparative analysis using linearized Least-Squares and Bayesian methods, J. Geodyn., 48, 331-339.

Rosat, S., Calvo, M. \& Lambert, S., 2016. Detailed analysis of diurnal tides and associated space nutation in the search for the Free Inner Core Nutation resonance, in International Association of Geodesy Symposia, doi:10.1007/1345_2016_224.

Savcenko, R. \& Bosch, W., 2011. EOT11a - a new tide model from MultiMission Altimetry, OSTST Meeting, October 19-21, San Diego.

Schuh, H. \& Behrend, D., 2012. VLBI: a fascinating technique for geodesy and astrometry, J. Geodyn., 61, 68-80.

Souchay, J., Loysel, B., Kinoshita, H. \& Folgueira, M., 1999. Corrections and new developments in rigid earth nutation theory: III. Final tables
"REN-2000" including crossed-nutation and spin-orbit coupling effects, Astron. Astrophys. Suppl. Ser., 135, 111-131.

Tamura, Y., 1987. A Harmonic development of the tide generating potential, Bull. Inf. Marées Terrestres, 99, 6813-6855.

Tarantola, A. \& Valette, B., 1982. Inverse Problems = Quest for Information, J. Geophys., 50, 159-170.

Wenzel, H.G., 1996. The nanogal software: Earth tide data processing package ETERNA 3.30, Bull. Inf. Marées Terr., 124, 9425-9439.

Zaske, J., Zürn, W. \& Wilhelm, H., 2000. NDFW analysis of Borehole Water Level Data from the Hot-Dry-Rock Test Site Soultz-Sous-Forêts, Bull. Inf. Marées Terr., 132, 10 241-10 270. 